\title{
Successful treatment with clarithromycin for a patient with rheumatoid arthritis
}

\author{
Masashi Ohe $^{1, *}$ and Toshiyuki Bohgaki ${ }^{2}$ \\ ${ }^{1}$ Department of General Medicine, Hokkaido Social Insurance Hospital, Sapporo, Japan \\ ${ }^{2}$ Department of Medicine II, Hokkaido University Graduate School of Medicine, Sapporo, Japan
}

\begin{abstract}
Macrolide antibiotics provide not only anti-bacterial activity but also anti-inflammatory effects. We report herein a case of rheumatoid arthritis (RA) successfully treated using clarithromycin (CAM), a macrolide, in expectation of its antiinflammatory effects. A 77-year-old woman suffering from RA, presented with exacerbation of arthralgia and articular swellings. Because she was suffering from non-tuberculous mycobacterium and could not receive biological agents, CAM was successfully added to prednisolone and a nonsteroidal anti-inflammatory drug which had been administered before exacerbation. The present case suggests that CAM treatment may be effective for RA.
\end{abstract}

Key Words: Rheumatoid arthritis, clarithromycin

\section{Introduction}

Macrolide antibiotics (Mac) such as erythromycin and clarithromycin (CAM) provide not only antibacterial activity but also anti-inflammatory effects. Regarding anti-inflammatory effects, successful treatments with Mac for diffuse panbronchiolitis, cystic fibrosis, chronic obstructive pulmonary disease, and bronchial asthma have been reported. Similarly, several recent studies have reported successful treatment of rheumatoid arthritis (RA) with CAM (1-3). RA is an inflammatory disease in which proinflammatory cytokines such as tumor necrosis factor (TNF)- $\alpha$ are associated with clinical features. We report here a case of uncontrolled RA accompanied by non-tuberculous mycobacterium (NTM) that was successfully treated using CAM in expectation of its antiinflammatory effects suppressing TNF- $\alpha$ (4).

\section{Case report}

A 77-year-old woman suffering from RA, presented with exacerbation of arthralgia and articular swellings. She had been diagnosed with $\mathrm{RA}$ at 67 years of age and had been administered salazosulfapyridine (SASP) and subsequently SASP in combination with prednisolone (PSL) at 3 $\mathrm{mg} /$ day. Her general condition including arthralgia and articular swellings had been stable under these treatments for 7 years; however, arthralgia and articular swellings had worsened. SASP was changed to methotrexate (MTX) at 6 $\mathrm{mg} /$ week, and her symptoms improved. Because liver dysfunction occurred as a side effect of MTX, the MTX was stopped. As a result, her symptoms worsened. As an alternative to MTX, tacrolimus (Tac) at $1.5 \mathrm{mg} /$ day was administered, and her symptoms improved again. Because renal dysfunction occurred as a side effect of Tac, the Tac was stopped. We then investigated whether or not the patient could receive biological agents such as TNF- $\alpha$ blocking agents. Hepatitis B surface antigen and anti-hepatitis $\mathrm{C}$ antibody were negative. Chest computed tomography (CT) revealed centrilobular opacities, dilated bronchus and patchy opacities in the right middle lobe and ground-glass opacities, patchy opacities and pleural thickness in the bilateral lower lobes (Figure 1). Mycobacterium avium was detected in bronchoalveolar lavage specimens from the right middle lobe. Therefore, the patient was diagnosed with NTM. As a result, she was determined to be unable to receive biological agents. Therefore, she was treated with PSL at $7.5 \mathrm{mg} /$ day and subsequently $10 \mathrm{mg} /$ day in combination with loxoprofen (LOX) at $180 \mathrm{mg} /$ day for her moderate arthralgia and articular swellings. On this visit, her C-reactive protein (CRP) level and disease activity score (DAS)28-CRP, which is 
defined as $0.56 \sqrt{ } \mathrm{TJC} 28+0.28 \sqrt{\mathrm{SJC}} 28+0.36 \mathrm{ln}$ $((\mathrm{CRP}(\mathrm{mg} / \mathrm{dL}) \times 10+1)+0.014$ General Health + 0.96, (TJC28: 28 Tender joint count; SJC28: 28 Swollen joint count; General Health: patient assessment of disease activity using a 100-mm visual analogue scale with $0=$ best, $100=$ worst) (5), were $2.65 \mathrm{mg} / \mathrm{dL}$ and 4.78 , respectively. Because the PSL dosage could not be increased any further, for fear of osteoporosis and exacerbation of NTM, CAM at $400 \mathrm{mg} /$ day was added to PSL and LOX in expectation of its anti-inflammatory effects after obtaining informed consent. Two weeks after this treatment, arthralgia and articular swellings began to improve. Sixteen weeks after starting this treatment, the arthralgia and articular swellings improved considerably, with CRP and DAS28-CRP decreasing to $0.73 \mathrm{mg} / \mathrm{dL}$ and 2.79 , respectively. The PSL dosage was gradually reduced to $6 \mathrm{mg} /$ day. The abnormal opacities on chest CT remained unchanged after CAM treatment.

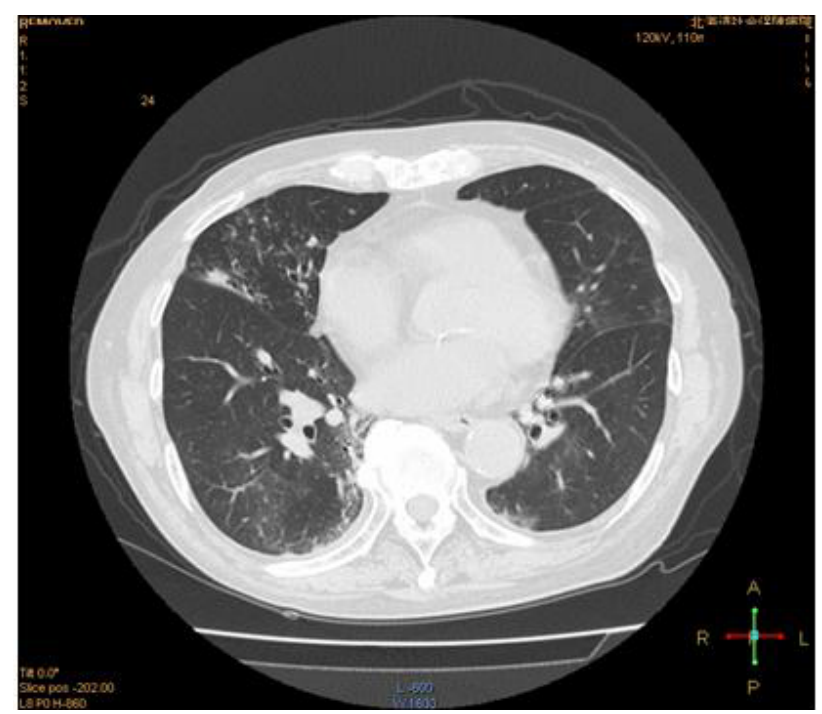

Fig. 1. Chest computed tomography showing centrilobular opacities, dilated bronchus and patchy opacities in the right middle lobe and ground-glass opacities, patchy opacities and pleural thickness in the bilateral lower lobes.

\section{Discussion}

The introductions of biological agents such as TNF- $\alpha$ blocking agents, namely, infliximab and etanercept resulted in a paradigm shift in the treatment of RA (6). Although these agents are efficacious against RA, serious adverse reactions (including infections) can arise. As a result, treatment guidelines for the use of these agents have been proposed (7). According to those guidelines, use of such agents is prohibited or not recommended for patients with current infections such as hepatitis B or NTM. Because the patient was suffering from NTM, she could not receive biological agents based on those guidelines. As an alternative, CAM at $400 \mathrm{mg} /$ day was successfully added. According to European League Against Rheumatism response criteria, CAM treatment achieved good response (5).

As stated above, Mac provide not only antibacterial activity but also anti-inflammatory effects. Regarding anti-inflammatory effects, Mac have been shown to affect several pathways of the inflammatory process such as the production of proinflammatory cytokines (1). In fact, CAM has been shown to inhibit the production of $\mathrm{TNF}_{-} \alpha$ (4), which is known to be associated with clinical features of RA. Mac also exert a substantial antiinflammatory influence through their antibacterial activity. For instance, Mac are active against periodontopathic bacteria, which are powerful stimulators of TNF- $\alpha$ and other proinflammatory cytokines in humans (1). Because Ogrendik hypothesized that oral anaerobic bacteria could play an important role in the pathogenesis of RA (8), the effectiveness of CAM for RA may be derived from its antibacterial activity as well as its anti-inflammatory effects. Regarding CAM treatment for RA, Ogrendik reported that treatment with CAM (500 mg/day for 6 months) improved the signs and symptoms of patients with early active RA (1). Saviola et al. (2) reported that CAM (500 mg twice per day for the first 10 days, followed by $250 \mathrm{mg}$ twice per day for the long term) could be beneficial in RA patients who were not responsive to, or could not tolerate diseasemodifying antirheumatic drugs (DMARDs). They also reported the efficacy of the addition of CAM (500 mg twice per day for the first 15 days, followed by $500 \mathrm{mg}$ per day for the remaining 15 days) to MTX and low-dose methyl-PSL in active RA (3). In the present case, a patient who had already received PSL was treated with CAM. Because Mac have steroid-sparing effects via their influence on corticosteroid metabolism (9), this may have also played a role (along with the abovementioned anti-inflammatory effects) in improving the signs and symptoms of RA. Based on these findings, CAM treatment may be effective and especially helpful for RA patients unable to receive biological agents, DMARDs, or increasing doses of PSL because of medical conditions.

Conflict of interest: No potential conflict of interest relevant to this article is reported. 


\section{References}

1. Ogrendik M. Effects of clarithromycin in patients with active rheumatoid arthritis. Curr Med Res Opin 2007; 23: 515-522.

2. Saviola G, Abdi Ali L, Rossini P, et al. Clarithromycin in rheumatoid arthritis patients not responsive to disease-modifying antirheumatic drugs: an open, uncontrolled pilot study. Clin Exp Rheumatol 2002; 20: 373-378.

3. Saviola G, Abdi-Ali L, Campostrini L, et al. Clarithromycin in rheumatoid arthritis: the addition to methotrexate and low-dose methylprednisolone induces a significant additive value--a 24-month single-blind pilot study. Rheumatol Int 2013; 33: 2833-2838.

4. Tkalcevic VI, Bosnjak B, Pasalic I, et al. The antiinflammatory activity of clarithromycin inhibits TNFalpha production and prolongs survival following lipopolysaccharide administration in mice. Int J Antimicrob Agents 2008; 32: 195-196.
5. Wells G, Becker JC, Teng J, et al. Validation of the 28-joint Disease Activity Score (DAS28) and European League Against Rheumatism response criteria based on C-reactive protein against disease progression in patients with rheumatoid arthritis, and comparison with the DAS28 based on erythrocyte sedimentation rate. Ann Rheum Dis 2009; 68: 954-960.

6. JungYO, Kim HA. Recent paradigm shifts in the diagnosis and treatment of rheumatoid arthritis. Korean J Intern Med 2012; 27: 378-387.

7. Koike R, Takeuchi T, Eguchi K, Miyasaka N. Update on the Japanese guidelines for the use of infliximab and etanercept in rheumatoid arthritis. Mod Rheumatol 2007; 17: 451-458.

8. Ogrendik M. Antibiotics for the treatment of rheumatoid arthritis. Int J Gen Med 2014; 7: 4347.

9. Rubin BK, Henke MO. Immunomodulatory activity and effectiveness of macrolides in chronic airway diseases. Chest 2004; 125: 70-78. 